PROCEEDINGS OF THE

AMERICAN MATHEMATICAL SOCIETY

Volume 140, Number 9, September 2012, Pages 3263-3273

S 0002-9939(2012)11180-3

Article electronically published on January 30, 2012

\title{
A NEW CHARACTERIZATION OF CONVEXITY IN FREE CARNOT GROUPS
}

\author{
ANDREA BONFIGLIOLI AND ERMANNO LANCONELLI
}

(Communicated by Matthew J. Gursky)

\begin{abstract}
A characterization of convex functions in $\mathbb{R}^{N}$ states that an upper semicontinuous function $u$ is convex if and only if $u(A x)$ is subharmonic (with respect to the usual Laplace operator) for every symmetric positive definite matrix $A$. The aim of this paper is to prove that an analogue of this result holds for free Carnot groups $\mathbb{G}$ when considering convexity in the viscosity sense. In the subelliptic context of Carnot groups, the linear maps $x \mapsto A x$ of the Euclidean case must be replaced by suitable group isomorphisms $x \mapsto T_{A}(x)$, whose differential preserves the first layer of the stratification of $\operatorname{Lie}(\mathbb{G})$.
\end{abstract}

\section{INTRODUCTION}

A characterization of convexity in $\mathbb{R}^{N}$ states that an upper semicontinuous (u.s.c., for short) function $u: \mathbb{R}^{N} \rightarrow[-\infty, \infty)$ is convex (in the classical sense) if and only if, for every positive definite matrix $A$, the function $x \mapsto u(A x)$ is subharmonic, with respect to the usual Laplace operator in $\mathbb{R}^{N}$ (see [20]).

The aim of this paper is to establish an analogue on Carnot groups $\mathbb{G}$ for $v$ convex functions, i.e., for functions which are convex in the viscosity sense first introduced by Lu, Manfredi and Stroffolini in [22] and by Juutinen, Lu, Manfredi and Stroffolini in 21. Roughly speaking, these authors call v-convex any function which is subharmonic with respect to every sub-Laplacian of the stratified group $\mathbb{G}$. On the other hand, if $\mathbb{G}$ is free, we know that every sub-Laplacian can be reduced to a single one via a "linear" change of variables; see [6].

These ideas have led us to show that, in the free setting, a u.s.c. function $u$ : $\mathbb{G} \rightarrow\left[-\infty, \infty\left[\right.\right.$ is v-convex if and only if a suitable family of functions $\left\{u \circ \Theta_{B}\right\}_{B}$ is subharmonic with respect to a fixed sub-Laplacian on $\mathbb{G}$. More precisely, if $m$ denotes the dimension of the horizontal layer $H$ of $\operatorname{Lie}(\mathbb{G})$ and $B$ varies over the cone of the positive definite $m \times m$ matrices, then $\Theta_{B}$ is the (unique) group automorphism of $\mathbb{G}$ whose differential coincides (when restricted to $H$ ) with the linear endomorphism of $H$ associated to $B$. See Theorem 3.8 below for the precise statement. We explicitly remark that the well-posedness of the above $\Theta_{B}$ is a consequence of the freeness of $\mathbb{G}$. In the Euclidean case $\mathbb{G}=\left(\mathbb{R}^{N},+\right)$, this gives back the classical result cited in the introduction, since, in this case, $\Theta_{B}$ coincides with the linear map associated to $B$.

The idea of characterizing the v-convexity in terms of a fixed sub-Laplacian is, moreover, in the spirit of a characterization given in [21, where it is proved that

Received by the editors October 13, 2010 and, in revised form, March 30, 2011

2000 Mathematics Subject Classification. Primary 31C05, 26B25, 43A80; Secondary 35J70.

(C)2012 American Mathematical Society 
$u$ is v-convex if and only if $\left(D_{\mathrm{h}}^{2} u\right)^{*} \geq 0$ in the viscosity sense. Here $\left(D_{\mathrm{h}}^{2} \phi\right)^{*}=$ $\left(\frac{1}{2}\left(X_{i} X_{j} \phi+X_{j} X_{i} \phi\right)\right)_{i, j}$ denotes the symmetrized horizontal second derivative matrix of $\phi \in C^{2}$, with respect to the fixed horizontal fram 11 \{ $\left\{X_{1}, \ldots, X_{m}\right\}$.

Our main argument here is the following one. Assuming any fixed sub-Laplacian $\mathcal{L}_{0}=\sum_{i=1}^{m} Z_{i}^{2}$ on $\mathbb{G}$, any other sub-Laplacian $\mathcal{L}$ on $\mathbb{G}$ is of the form $\mathcal{L}=\sum_{i=1}^{m} X_{i}^{2}$, where $X_{i}=\sum_{j=1}^{m} b_{i, j} Z_{j}$ (for $i=1, \ldots, m$ ) and $B=\left(b_{i, j}\right)$ is a non-singular matrix. In other words, setting $H:=\operatorname{span}\left\{Z_{1}, \ldots, Z_{m}\right\}$, the system of vector fields $\left\{X_{1}, \ldots, X_{m}\right\}$ is obtained from $\mathcal{Z}:=\left\{Z_{1}, \ldots, Z_{m}\right\}$ via the linear isomorphism $\varphi_{B}: H \rightarrow H$ naturally associated (w.r.t. the basis $\mathcal{Z}$ ) to the matrix $B^{T}$. Since $\operatorname{Lie}(\mathbb{G})$ is free and it is Lie-generated by $\mathcal{Z}, \varphi_{B}$ extends to a unique Lie-algebra isomorphism of $\operatorname{Lie}(\mathbb{G})$, say $\widehat{\varphi}_{B}$. If we denote by $\Theta_{B}: \mathbb{G} \rightarrow \mathbb{G}$ the (unique) isomorphism of the Lie group $\mathbb{G}$ whose differential is $\widehat{\varphi}_{B}$, then it is not difficult to recognize that, in the new coordinates defined by $x \mapsto y=\Theta_{B}(x)$, the sub-Laplacian $\mathcal{L}_{0}$ turns into $\mathcal{L}$, or, more precisely, $(\mathcal{L} u) \circ \Theta_{B}=\mathcal{L}_{0}\left(u \circ \Theta_{B}\right)$, for every smooth $u$ on $\mathbb{G}$ (see Lemma 3.11).

Our main task is then to show that the $\mathcal{L}$-subharmonicity of $u$ is equivalent to the $\mathcal{L}_{0}$-subharmonicity of $u \circ \Theta_{B}$. Whereas this is easily seen when $u$ is of class $C^{2}$, for arbitrary u.s.c. functions $u$ this is more concealed. We shall prove it by making use of a suitable submean characterization of subharmonic functions on Carnot groups, as given in our previous paper [3]. We shall see that the mean-integral operators $M^{\mathcal{L}}$ and $M^{\mathcal{L}_{0}}$ related, respectively, to the sub-Laplacians $\mathcal{L}$ and $\mathcal{L}_{0}$ are (roughly speaking) $\Theta_{B}$-related, i.e., $M^{\mathcal{L}}(u) \circ \Theta_{B}=M^{\mathcal{L}_{0}}\left(u \circ \Theta_{B}\right)$ for every u.s.c. function $u$ (see Lemma 3.4 for the precise statement). Since the mean-integral operators on Carnot groups which are different from the Euclidean one always involve nonidentically 1 density kernels, this result seems to have some interest in its own right. Moreover, we hope that the above idea of changing coordinates via the maps $\Theta_{B}$ may be fruitful, in the context of convexity, as it has already been in other contexts (see [4, 6]).

Before closing the introduction, we would like to recall some works on convexity in Carnot groups. Due to the relevance of the notion of convexity in theoretical and applied areas of mathematics, several notions of convexity have been recently proposed in the context of Carnot groups. The papers by Danielli, Garofalo and Nhieu [10] and by Lu, Manfredi and Stroffolini [22] opened the way for appropriate definitions of convexity in this context: they respectively introduced the classes of h-convex (horizontally convex) and v-convex (viscosity convex) functions. On the Heisenberg groups, these notions coincide (Balogh and Rickly [1]), whereas, generally, the v-convex functions are the u.s.c.h-convex functions (see Magnani [23, Wang [32]). In this paper we are concerned with the notion of v-convexity.

Along with the mentioned references, contributions to the understanding of convexity in stratified groups come from many authors. The problem of fine regularity for convex functions has especially been investigated. See Capogna and Maldonado [7] Capogna, Pauls, and Tyson [8]; Danielli, Garofalo, Nhieu, and Tournier [11; Garofalo [14; Garofalo and Tournier [15]; Gutiérrez and Montanari [17, 18; Juutinen, Lu, Manfredi, and Stroffolini [21]; Monti and Rickly [24, Rickly [25]; Sun and Yang [28, 29]. (For the notion of "r-convex" function in Carnot groups, see Dah-Yan [9] and Sun and Yang [26, 27.)

\footnotetext{
${ }^{1}$ This is any basis of the first layer of the stratification of the Lie algebra of $\mathbb{G}$.
} 


\section{Notation AND DEFinitions}

A Carnot group is a couple $\left(G, V_{1}\right)$ where $(G, \cdot)$ is a connected and simply connected (real) Lie group and $V_{1}$ is a subspace of the Lie algebra $\operatorname{Lie}(G)$ of $G$ such that $\operatorname{Lie}(G)=V_{1} \oplus \cdots \oplus V_{r}$, where $V_{i}=\left[V_{1}, V_{i-1}\right]$, for $2 \leq i \leq r$ and $\left[V_{1}, V_{r}\right]=\{0\}$. Throughout the paper, we set the positions

$$
H:=V_{1}, \quad m:=\operatorname{dim}\left(V_{1}\right) .
$$

Vector fields in $H$ are usually called horizontal. The integer $Q:=\sum_{i=1}^{r} i \operatorname{dim}\left(V_{i}\right)$ is called the homogeneous dimension of $\mathbb{G}$. Throughout this paper we assume that $Q \geq 3$. A sub-Laplacian on $\mathbb{G}$ is a differential operator of the form $\sum_{i=1}^{m} X_{i}^{2}$, where $\left\{X_{1}, \ldots, X_{m}\right\}$ is any basis of $H$.

We next recall the definition of free Carnot group. To this aim we first need the notion of free nilpotent Lie algebra $\mathfrak{f}_{m, r}$ of step $r$ and $m$ generators. By definition, $\mathfrak{f}_{m, r}$ is the unique (up to isomorphism) nilpotent Lie algebra of step $r$ Lie-generated by $m(\geq 2)$ of its elements, say $F_{1}, \ldots, F_{m}$, with the following property: for every Lie algebra $\mathfrak{n}$, nilpotent of step $\leq r$, and for every map $L$ from $\left\{F_{1}, \ldots, F_{m}\right\}$ to $\mathfrak{n}$, there exists a (unique) Lie algebra morphism from $\mathfrak{f}_{m, r}$ to $\mathfrak{n}$ extending $L$. The construction of such a Lie algebra $\mathfrak{f}_{m, r}$ is classical (see, e.g., 30, 31]; the reader is also referred to [19, 16] for the construction of a basis for $\mathfrak{f}_{m, r}$ ). We say that a Carnot group $G$ is a free Carnot group if its Lie algebra is isomorphic to $\mathfrak{f}_{m, r}$, for some $m$ and $r$. Notice that, in this case, $m$ necessarily equals the dimension of $H$ (as in (2.1) ) and $r$ is the step of nilpotency of $G$.

In order to simplify computations, we fix suitable coordinates on $G$. Since a Carnot group is nilpotent, the exponential map $\operatorname{Exp}: \operatorname{Lie}(G) \rightarrow G$ is an (analytic) diffeomorphism. If the $V_{i}$ 's are as in the introduction of this section, we fix a basis $\mathcal{E}$ for $\operatorname{Lie}(G)$ which is "adapted" to the stratification, i.e.,

$$
\mathcal{E}=\left\{E_{1}^{(1)}, \ldots, E_{m}^{(1)} ; E_{1}^{(2)}, \ldots, E_{N_{2}}^{(2)} ; \ldots ; E_{1}^{(r)}, \ldots, E_{N_{r}}^{(r)}\right\}=:\left\{E_{1}, \ldots, E_{N}\right\},
$$

where (for any $i=1, \ldots, r) N_{i}:=\operatorname{dim}\left(V_{i}\right), N=m+N_{2}+\cdots+N_{r}$ and $E_{1}^{(i)}, \ldots, E_{N_{i}}^{(i)}$ is a basis for $V_{i}$. Via the exponential map and via this choice of basis for $\operatorname{Lie}(G)$, we can identify $G$ to $\mathbb{R}^{N}$. This amounts to fixing a global coordinate system

$$
\varphi: G \rightarrow \mathbb{R}^{N}, \quad g \mapsto \varphi(g)=:\left(x_{1}, \ldots, x_{N}\right),
$$

where $\varphi^{-1}\left(x_{1}, \ldots, x_{N}\right)=\operatorname{Exp}\left(x_{1} E_{1}+\cdots+x_{N} E_{N}\right)$. This identification is particularly useful since the Haar measure on $G$ simply boils down to the Lebesgue measure on $\mathbb{R}^{N}$. Also, the multiplication · on $G$ "pushes forward" to the following composition law on $\mathbb{R}^{N}$ :

$$
x * y:=\varphi\left(\varphi^{-1}(x) \cdot \varphi^{-1}(y)\right), \quad x, y \in \mathbb{R}^{N} .
$$

(Due to the definition of $\varphi$, we recognize that the analogue of $*$ on $\operatorname{Lie}(\mathbb{G})$ is simply the Campbell-Baker-Hausdorff-Dynkin multiplication.) Form now on, we will tacitly identify $(G, \cdot)$ with the group $\mathbb{G}=\left(\mathbb{R}^{N}, *\right)$. We further equip the latter group with the family of non-isotropic "dilations"

$$
\begin{aligned}
\delta_{\lambda}: \mathbb{R}^{N} & \rightarrow \mathbb{R}^{N}, \quad \delta_{\lambda}\left(x_{1}, \ldots, x_{N}\right) \\
& :=\left(\lambda x_{1}, \ldots, \lambda x_{m} ; \lambda^{2} x_{1}^{(2)}, \ldots, \lambda^{2} x_{N_{2}}^{(2)} ; \ldots ; \lambda^{r} x_{1}^{(r)}, \ldots, \lambda^{r} x_{N_{r}}^{(r)}\right) .
\end{aligned}
$$

Here we applied the obvious notation suggested by (2.2). Following the definition of [5, $\S 1.4]$, we say that $\left(\mathbb{R}^{N}, *, \delta_{\lambda}\right)$ is a homogeneous Carnot group of step $r$ and $m$ generators. As shown in $[5$, a selected basis for $\operatorname{Lie}(\mathbb{G})$ is given by the vector fields 
$Z_{1}, \ldots, Z_{N}$, whose component functions (w.r.t. $\partial_{x_{1}}, \ldots, \partial_{x_{N}}$ ) are given by the $N$ column vectors of $\mathcal{J}_{\tau_{x}}(0)$, the Jacobian matrix at 0 of the left translations $\tau_{x}(y)=$ $x * y$. Then (see [5, Proposition 2.2.22]) the exponential map of $\mathbb{G}$ is given by

$$
\operatorname{Exp}: \operatorname{Lie}(\mathbb{G}) \rightarrow \mathbb{G}, \quad x_{1} Z_{1}+\cdots+x_{N} Z_{N} \mapsto\left(x_{1}, \ldots, x_{N}\right) .
$$

In the sequel, we shall consider the following "canonical" sub-Laplacian on $\mathbb{G}$ :

$$
\Delta_{\mathbb{G}}:=\sum_{i=1}^{m} Z_{i}^{2} .
$$

(This choice, along with the above choice of coordinates, is obviously immaterial, and it is only meant to simplify computations throughout and to fix the notation.) Moreover, we denote by $\mathcal{M}$ the vector space of the real $m \times m$ matrices and we set

$$
\mathcal{A}:=\{A \in \mathcal{M} \mid A \text { is symmetric and positive definite }\} .
$$

For every $A=\left(a_{i, j}\right)_{i, j \leq m}$ in $\mathcal{A}$, we consider the differential operator

$$
\mathcal{L}_{A}:=\sum_{i, j=1}^{m} a_{i, j} Z_{i} Z_{j} .
$$

It is easily seen that the family $\left\{\mathcal{L}_{A}\right\}_{A \in \mathcal{A}}$ is precisely the family of all sub-Laplacians on $\mathbb{G}$. Indeed, a sub-Laplacian on $\mathbb{G}$ is of the form $\mathcal{L}=\sum_{i=1}^{m} X_{i}^{2}$, where

$$
X_{i}=\sum_{j=1}^{m} b_{i, j} Z_{j}, \quad i=1, \ldots, m,
$$

where $B=\left(b_{i, j}\right)_{i, j \leq m} \in \mathcal{M}$ is non-singular, so that $\mathcal{L}=\mathcal{L}_{A}$ with $A=B^{T} \cdot B$ (which obviously belongs to $\mathcal{A}$ ). Vice versa, if $A \in \mathcal{A}$ and if $B \in \mathcal{M}$ is any non-singular matrix satisfying $A=B^{T} \cdot B$ (for example, $B=A^{1 / 2}$, the symmetric positive definite square root of $A$ ), we have $\mathcal{L}_{A}=\sum_{i=1}^{m} X_{i}^{2}$ where the $X_{i}$ 's are as in (2.8), whence $\mathcal{L}_{A}$ is indeed a sub-Laplacian on $\mathbb{G}$. This is a consequence of the following computation, to which we will return later in this paper:

$$
\begin{aligned}
\sum_{i=1}^{m} X_{i}^{2} & \stackrel{(2.8)}{=} \sum_{j, k=1}^{m}\left(\sum_{i=1}^{m} b_{i, j} b_{i, k}\right) X_{j} X_{k}=\sum_{j, k=1}^{m}\left(B^{T} \cdot B\right)_{j, k} X_{j} X_{k} \\
& =\sum_{j, k=1}^{m} a_{j, k} X_{j} X_{k}=\mathcal{L}_{A} .
\end{aligned}
$$

We next turn to fundamental solutions. If $\mathcal{L}$ is any sub-Laplacian on $\mathbb{G}$, we say that $\Gamma$ is the fundamental solution of $\mathcal{L}$ if the following facts hold:

(i) $\Gamma: \mathbb{R}^{N} \rightarrow \mathbb{R} \cup\{\infty\}$ and $\Gamma \in C^{\infty}\left(\mathbb{R}^{N} \backslash\{0\}, \mathbb{R}\right)$;

(ii) $\Gamma$ vanishes at infinity;

(iii) $\Gamma \in L_{\text {loc }}^{1}\left(\mathbb{R}^{N}\right)$ and, in the weak sense of distributions, $-\mathcal{L} \Gamma$ is the Dirac measure supported at $\{0\}$.

By the weak maximum principle for $\mathcal{L}$, it is easily seen that the above $\Gamma$ (whose existence follows, e.g., from the results in [12]) is unique. In the sequel, we set

$$
d_{\mathcal{L}}:=\Gamma^{1 /(2-Q)}
$$

(with the convention $d_{\mathcal{L}}(0)=0$ ) and we say that $d_{\mathcal{L}}$ is the $\mathcal{L}$-gauge. The definition of $d_{\mathcal{L}}$ is well-posed since $\Gamma(x)>0$ for every $x \neq 0$, and it turns out that $d_{\mathcal{L}} \in C\left(\mathbb{R}^{N}\right) \cap$ $C^{\infty}\left(\mathbb{R}^{N} \backslash\{0\}\right)$ and that $d_{\mathcal{L}}\left(x^{-1}\right)=d_{\mathcal{L}}(x), d_{\mathcal{L}}\left(\delta_{\lambda}(x)\right)=\lambda d_{\mathcal{L}}(x)$, for every $\lambda>0$ and 
$x \in \mathbb{R}^{N}$. For a general Carnot group $\mathbb{G}$, we cannot expect the fundamental solutions of different sub-Laplacians on $\mathbb{G}$ to have much in common. Instead, for free Carnot groups, all fundamental solutions are related to the fundamental solution of $\Delta_{\mathbb{G}}$ by a strikingly simple formula (see Section 3).

We next turn to the definition of $\mathcal{L}$-subharmonicity. Among plenty of possible equivalent definitions (see [3] and [5, Chapter 8]), we choose the following one, since we shall employ the mean integral operator $M_{r}$ below, again in this paper. Let $\mathbb{G}$ be a Carnot group, $\mathcal{L}=\sum_{i=1}^{m} X_{i}^{2}$ a sub-Laplacian on $\mathbb{G}, \Gamma$ its fundamental solution and $d_{\mathcal{L}}$ the relevant $\mathcal{L}$-gauge. If $\Omega \subseteq \mathbb{G}$ is open, $u: \Omega \rightarrow[-\infty, \infty)$ is an upper semi-continuous (u.s.c., in short) function, and $x \in \Omega$ and $r>0$ are such that

$$
D_{\mathcal{L}}(x, r):=\left\{y \in \mathbb{R}^{N}: d_{\mathcal{L}}\left(x^{-1} * y\right)<r\right\}
$$

has closure contained in $\Omega$, we set

$$
M_{r}^{\mathcal{L}}(u)(x):=\frac{Q(Q-2)}{r^{Q}} \int_{d_{\mathcal{L}}\left(x^{-1} * y\right)<r} u(y) \Psi_{\mathcal{L}}\left(x^{-1} * y\right) \mathrm{d} y,
$$

where $\Psi_{\mathcal{L}}:=\sum_{i=1}^{m}\left|X_{i} d_{\mathcal{L}}\right|^{2}$.

With the above notation, we say that a u.s.c. function $u: \Omega \rightarrow[-\infty, \infty)$ is $\mathcal{L}$ subharmonic in $\Omega$ if

$$
u(x) \leq M_{r}^{\mathcal{L}}(u)(x), \quad \text { whenever } \overline{D_{\mathcal{L}}(x, r)} \subset \Omega .
$$

Finally, we recall the notion of v-convexity (convexity in the viscosity sense; see $22]$ ). Given an open set $\Omega \subseteq \mathbb{G}$, a u.s.c. function $u: \Omega \rightarrow[-\infty, \infty)$ is $v$-convex in $\Omega$ if $u$ is $\mathcal{L}$-subharmonic with respect to all sub-Laplacians $\mathcal{L}$ on $\mathbb{G}$. As we said in the introduction, this definition can be compared to other remarkable definitions available in the literature (as that of horizontal convexity; see [10]). Thanks to the remarks made a few paragraphs above, we explicitly observe that $u$ is $\mathrm{v}$-convex if and only if it is $\mathcal{L}_{A}$-subharmonic for every $A \in \mathcal{A}$, where $\mathcal{L}_{A}$ is as in (2.7).

\section{A NEW CHARACTERIZATION OF V-CONVEXITY IN FREE GROUPS}

Throughout the sequel, $\mathbb{G}=\left(\mathbb{R}^{N}, *, \delta_{\lambda}\right)$ is a fixed free (homogenous) Carnot group and the notation in Section 2 is tacitly employed. We begin with the following central result. It improves (both in the statement and in the proof) Theorem 16.1.2 of [5] and Theorem 2.2 of $[\underline{6}$.

Lemma 3.1. Let $A \in \mathcal{A}$. There exists a group isomorphism $T_{A}: \mathbb{G} \rightarrow \mathbb{G}$ such that

$$
\left(\mathcal{L}_{A} u\right) \circ T_{A}=\Delta_{\mathbb{G}}\left(u \circ T_{A}\right), \quad \forall u \in C^{\infty}(\mathbb{G}, \mathbb{R}) .
$$

More precisely, $T_{A}$ can be constructed as follows. If $B=\left(b_{i, j}\right)_{i, j \leq m}$ is any (nonsingular) real matrix satisfying $A=B^{T} \cdot B$ (for example $B=A^{1 / 2}$ ) and if we denote by $\left\{X_{1}, \ldots, X_{m}\right\}$ the basis of $H$ defined in (2.8), then

$$
T_{A}=\operatorname{Exp} \circ \varphi_{B} \circ \log ,
$$

where $\varphi_{B}: \operatorname{Lie}(\mathbb{G}) \rightarrow \operatorname{Lie}(\mathbb{G})$ is the unique Lie-algebra isomorphism mapping $Z_{i}$ into $X_{i}$, for any $i=1, \ldots, m$.

Actually, with respect to the logarithmic coordinates previously fixed on $\mathbb{G}, T_{A}$ is linear and it coincides with the linear map related to the matrix representing $\left(\right.$ w.r.t. the basis $\left\{Z_{1}, \ldots, Z_{N}\right\}$ of $\operatorname{Lie}(\mathbb{G})$ ) the linear function $\varphi_{B}: \operatorname{Lie}(\mathbb{G}) \rightarrow \operatorname{Lie}(\mathbb{G})$. 
Remark 3.2. The notation $T_{A}=\operatorname{Exp} \circ \varphi_{B} \circ \log$ may be somewhat misleading: in fact, given $A \in \mathcal{A}$ there may exist many matrices $B \in \mathcal{M}$ such that $A=B^{T} \cdot B$. In the sequel, given $A \in \mathcal{A}$, when we write $T_{A}$ we mean any map of the form Exp $\circ \varphi_{B} \circ \log$, where $B \in \mathcal{M}$ is any matrix satisfying $A=B^{T} \cdot B$, whereas $\varphi_{B}$ is the unique Lie-algebra morphism described in the assertion of Lemma 3.1. We hope that the reader may accept this little abuse of notation up until the statement of our main result, Theorem 3.8 below, where all will be made unambiguous.

Proof. Let $A \in \mathcal{A}$ and let $B=\left(b_{i, j}\right)_{i, j \leq m} \in \mathcal{M}$ satisfy $A=B^{T} \cdot B$. Notice that $B$ is non-singular. By definition of Carnot group, the system of vector fields $\mathcal{Z}=\left\{Z_{1}, \ldots, Z_{m}\right\}$ Lie-generates $\mathfrak{g}:=\operatorname{Lie}(\mathbb{G})$. Thus, if we set

$$
X_{i}:=\sum_{j=1}^{m} b_{i, j} Z_{j}, \quad i=1, \ldots, m,
$$

$\mathfrak{g}$ being a free nilpotent Lie algebra, there exists a unique Lie-algebra morphism $\varphi_{B}: \mathfrak{g} \rightarrow \mathfrak{g}$ extending the map on $\mathcal{Z}$ defined by

$$
Z_{i} \mapsto X_{i}, \quad i=1, \ldots, m .
$$

It is easily seen that $\varphi_{B}$ is a Lie-algebra isomorphism.

Since $\mathbb{G}$ is simply connected, there exists a unique Lie-group morphism $T_{A}: \mathbb{G} \rightarrow$ $\mathbb{G}$ whose differential is $\varphi_{B}$. It is not difficult to see that this is precisely given by the position $T_{A}:=\operatorname{Exp} \circ \varphi_{B} \circ \log$. Notice that $T_{A}$ is an isomorphism since its differential $\varphi_{B}$ is invertible. If we show that

$$
\left(X_{i} u\right) \circ T_{A}=Z_{i}\left(u \circ T_{A}\right), \quad \text { for all } u \in C^{\infty}(\mathbb{G}, \mathbb{R}) \text { and all } i \in\{1, \ldots, m\},
$$

then (3.1) will follow, since $\Delta_{\mathbb{G}}=\sum_{i=1}^{m} Z_{i}^{2}$ and $\mathcal{L}_{A}=\sum_{i=1}^{m} X_{i}^{2}$ (the latter being a direct consequence of $A=B^{T} \cdot B$ and the definition of the $X_{i}$ 's). Now, (3.3) is a consequence of the following computation: for every smooth $u$ and any $x \in \mathbb{G}$,

$$
\begin{aligned}
Z_{i}\left(u \circ T_{A}\right)(x) & =\left.Z_{i}\right|_{x}\left(u \circ T_{A}\right)=\left(\mathrm{d}_{x} T_{A}\left(\left.Z_{i}\right|_{x}\right)\right) u=\left.\left(\mathrm{d} T_{A} Z_{i}\right)\right|_{T_{A}(x)} u \\
& =\left.X_{i}\right|_{T_{A}(x)} u=\left(X_{i} u\right)\left(T_{A}(x)\right) .
\end{aligned}
$$

Here we applied the fact that $\mathrm{d} T_{A}=\varphi_{B}, \varphi_{B}\left(Z_{i}\right)=X_{i}$ and the properties of differentials. The last assertion of the statement follows by collecting together the very definition of $T_{A}$ in (3.2) and the normalization resulting from our previous choice of coordinates (see (2.4)). This ends the proof.

Lemma 3.3. Let $A \in \mathcal{A}$. Let $T_{A}: \mathbb{G} \rightarrow \mathbb{G}$ be the group isomorphism as defined by (3.2) of Lemma 3.1, where $B \in \mathcal{M}$ is any matrix satisfying $A=B^{T} \cdot B$.

Let $\Gamma_{A}$ and $\Gamma$ denote respectively the fundamental solutions of the sub-Laplacians $\mathcal{L}_{A}$ and $\Delta_{\mathbb{G}}$. Then, there exists a constant $c_{A}>0$ such that

$$
\Gamma_{A}=c_{A} \cdot \Gamma \circ T_{A}^{-1}, \quad \text { on } \mathbb{G} .
$$

As a consequence, the $\mathcal{L}_{A^{-} \text {-gauge }} d_{\mathcal{L}_{A}}$ is given by

$$
d_{\mathcal{L}_{A}}=c_{A}^{1 /(2-Q)} \cdot d_{\Delta_{\mathbb{G}}} \circ T_{A}^{-1},
$$

where $d_{\Delta_{\mathbb{G}}}$ is the $\Delta_{\mathbb{G}}$-gauge. 
Proof. Let all the notation in the assertion hold. By the last statement of Lemma 3.1, the Jacobian matrix $\mathcal{J}_{T_{A}}(x)$ is independent of $x$. We let

$$
c_{A}:=1 /\left|\operatorname{det}\left(\mathcal{J}_{T_{A}}\right)(x)\right| .
$$

We are left to show that $\Gamma_{A}:=c_{A} \cdot \Gamma \circ T_{A}^{-1}$ is the fundamental solution of the sub-Laplacian $\mathcal{L}_{A}$. By definition, this amounts to proving the following facts:

(i) It holds that $\Gamma_{A}: \mathbb{R}^{N} \rightarrow \mathbb{R} \cup\{\infty\}$ and $\Gamma_{A} \in C^{\infty}\left(\mathbb{R}^{N} \backslash\{0\}, \mathbb{R}\right)$; this is obviously true, thanks to the analogous properties of $\Gamma$ and the fact that $T_{A}^{-1}$ is smooth, injective and null only at $x=0$.

(ii) $\Gamma_{A}$ vanishes at infinity; this derives from the analogous property of $\Gamma$ and the fact that $\lim _{|x| \rightarrow \infty}\left|T_{A}^{-1}(x)\right|=\infty, T_{A}^{-1}$ being a non-singular linear map.

(iii) $\Gamma_{A} \in L_{\text {loc }}^{1}\left(\mathbb{R}^{N}\right)$ and, in the weak sense of distributions, $-\mathcal{L}_{A} \Gamma_{A}$ is the Dirac measure supported at $\{0\}$; we only prove the last assertion since the $L_{\text {loc }}^{1}$ property is an obvious consequence of $\Gamma \in L_{\text {loc }}^{1}\left(\mathbb{R}^{N}\right)$.

To this aim, let $\varphi \in C_{0}^{\infty}\left(\mathbb{R}^{N}, \mathbb{R}\right)$. Then we have2

$$
\begin{aligned}
& \left.\int \Gamma_{A} \mathcal{L}_{A} \varphi=c_{A} \int \Gamma\left(T_{A}^{-1}(x)\right) \mathcal{L}_{A} \varphi(x) \mathrm{d} x \quad \text { (substituting } x=T_{A}(y)\right) \\
& =c_{A} \int \Gamma(y)\left(\mathcal{L}_{A} \varphi\right)\left(T_{A}(y)\right)\left|\operatorname{det}\left(\mathcal{J}_{T_{A}}\right)(y)\right| \mathrm{d} y
\end{aligned}
$$

(recall that $T_{A}$ is linear and use the definition of $c_{A}$ )

$$
\begin{aligned}
& =\int \Gamma(y)\left(\mathcal{L}_{A} \varphi\right)\left(T_{A}(y)\right) \mathrm{d} y \quad(\text { apply (3.1) }) \\
& =\int \Gamma(y) \Delta_{\mathbb{G}}\left(\varphi \circ T_{A}\right)(y) \mathrm{d} y=-\left(\varphi \circ T_{A}\right)(0)=-\varphi(0) .
\end{aligned}
$$

In the second-to-last equality we invoked the fact that $\Gamma$ is the fundamental solution of $\Delta_{\mathbb{G}}$ (and the fact that $\varphi \circ T_{A} \in C_{0}^{\infty}$ ). The last equality is a consequence of $T_{A}(0)=0$. This completes the proof.

Lemma 3.4. Let $A \in \mathcal{A}$. Let $T_{A}: \mathbb{G} \rightarrow \mathbb{G}$ be the group isomorphism as defined by (3.2) of Lemma 3.1, where $B \in \mathcal{M}$ is any matrix satisfying $A=B^{T} \cdot B$.

Consider the mean-integral operator defined in (2.9). Then for any u.s.c. function $u: \mathbb{G} \rightarrow[-\infty, \infty)$ and for every $r>0$ and $x \in \mathbb{G}$, we have

$$
M_{r}^{\mathcal{L}_{A}}(u)(x)=M_{\widetilde{r}}^{\Delta_{G}}\left(u \circ T_{A}\right)\left(T_{A}^{-1}(x)\right), \quad \text { with } \widetilde{r}=r c_{A}^{1 /(Q-2)} .
$$

Here, as usual, $c_{A}=1 /\left|\operatorname{det}\left(\mathcal{J}_{T_{A}}\right)(0)\right|$.

Proof. For the sake of brevity, we drop any prefix or suffix ' $\Delta_{\mathbb{G}}$ '. Let $A=B^{T} \cdot B$ and let $T_{A}=\operatorname{Exp} \circ \varphi_{B} \circ \log$ be as constructed in the proof of Lemma 3.1 Then $\mathcal{L}_{A}=\sum_{i=1}^{m} X_{i}^{2}$, where the $X_{i}$ 's are as in (2.8). Consequently, the relevant kernel $\Psi_{\mathcal{L}_{A}}$ in (2.9) is given by the following computation (see also Lemma 3.3):

$$
\begin{gathered}
\Psi_{\mathcal{L}_{A}}=\sum_{i=1}^{m}\left|X_{i} d_{\mathcal{L}_{A}}\right|^{2} \stackrel{(3.5)}{=} c_{A}^{2 /(2-Q)} \sum_{i=1}^{m}\left|X_{i}\left(d \circ T_{A}^{-1}\right)\right|^{2} \\
\stackrel{(3.3)}{=} c_{A}^{2 /(2-Q)} \sum_{i=1}^{m}\left|\left(Z_{i} d\right) \circ T_{A}^{-1}\right|^{2}=c_{A}^{2 /(2-Q)} \cdot \Psi \circ T_{A}^{-1} .
\end{gathered}
$$

\footnotetext{
${ }^{2}$ Here we implicitly use the fact that any sub-Laplacian is selfadjoint, since the formal adjoint of any field $X$ in $H$ equals $-X$ (see, e.g., [5] $\$ 1.5]$ ).
} 
Here, for brevity, $d$ is the $\Delta_{\mathbb{G}^{-}}$gauge, where $\Delta_{\mathbb{G}}=\sum_{i=1}^{m} Z_{i}^{2}$. We next write the set $D_{\mathcal{L}_{A}}$ in terms of $d$ : since $T_{A}^{-1}$ is a group morphism, one has

$$
d_{\mathcal{L}_{A}}\left(x^{-1} * y\right) \stackrel{\sqrt{3.5}}{=} c_{A}^{1 /(2-Q)} \cdot d\left(T_{A}^{-1}\left(x^{-1} * y\right)\right)=c_{A}^{1 /(2-Q)} \cdot d\left(\left(T_{A}^{-1}(x)\right)^{-1} * T_{A}^{-1}(y)\right) .
$$

This gives $D_{\mathcal{L}_{A}}(x, r)=T_{A}\left(D\left(T_{A}^{-1}(x), r c_{A}^{1 /(Q-2)}\right)\right)$. Summing up, we infer

$$
\begin{aligned}
& M_{r}^{\mathcal{L}_{A}}(u)(x) \stackrel{(2.9)}{=} \frac{Q(Q-2)}{r^{Q}} \int_{D_{\mathcal{L}_{A}}(x, r)} u(y) \Psi_{\mathcal{L}_{A}}\left(x^{-1} * y\right) \mathrm{d} y \\
& =\frac{Q(Q-2)}{r^{Q}} \int_{T_{A}\left(D\left(T_{A}^{-1}(x), r c_{A}^{1 /(Q-2)}\right)\right)} u(y) c_{A}^{2 /(2-Q)} \cdot \Psi\left(\left(T_{A}^{-1}(x)\right)^{-1} * T_{A}^{-1}(y)\right) \mathrm{d} y
\end{aligned}
$$

(use the substitution $y=T_{A}(z)$ and recall that $c_{A}=1 /\left|\operatorname{det} \mathcal{J}_{T_{A}}\right|$ )

$$
\begin{aligned}
& =\frac{Q(Q-2)}{r^{Q}} \int_{D\left(T_{A}^{-1}(x), r c_{A}^{1 /(Q-2)}\right)} u\left(T_{A}(z)\right) c_{A}^{2 /(2-Q)} \cdot \Psi\left(\left(T_{A}^{-1}(x)\right)^{-1} * z\right) c_{A}^{-1} \mathrm{~d} z \\
& =\frac{Q(Q-2)}{\widetilde{r}^{Q}} \int_{D(\widetilde{x}, \widetilde{r})} u\left(T_{A}(z)\right) \Psi\left(\widetilde{x}^{-1} * z\right) \mathrm{d} z=M_{\widetilde{r}}\left(u \circ T_{A}\right)(\widetilde{x}),
\end{aligned}
$$

where we have set $\widetilde{x}:=T_{A}^{-1}(x)$ and $\widetilde{r}=r c_{A}^{1 /(Q-2)}$. This is precisely (3.6).

We are ready for the following characterization of subharmonicity on a free group $\mathbb{G}$. (Obviously, mutatis mutandis, one can state an analogous characterization for subharmonicity on an arbitrary open set of the free group $\mathbb{G}$.)

Proposition 3.5. Let $\mathbb{G}$ be a free Carnot group. Let $u: \mathbb{G} \rightarrow[-\infty, \infty)$ be upper semicontinuous. Also let $A \in \mathcal{A}$ and let $\mathcal{L}_{A}$ be the sub-Laplacian defined in (2.7).

Then, $u$ is $\mathcal{L}_{A}$-subharmonic if and only if $u \circ T_{A}$ is $\Delta_{\mathbb{G}}$-subharmonic, where $T_{A}$ is any isomorphism of the form (3.2) (B being any matrix such that $A=B^{T} \cdot B$ ).

Remark 3.6. More precisely, we have two slightly stronger results (it being understood that the notation of the assertion holds):

(1) if $u$ is $\mathcal{L}_{A^{-}}$-subharmonic, then $u \circ T_{A}$ is $\Delta_{\mathbb{G}}$-subharmonic for every $B$ such that $A=B^{T} \cdot B$;

(2) if there exists at least one $B$ satisfying $A=B^{T} \cdot B$ for which $u \circ T_{A}$ is $\Delta_{\mathbb{G}}$-subharmonic, then $u$ is $\mathcal{L}_{A}$-subharmonic.

Proof of Proposition 3.5. Suppose that the u.s.c. function $u$ is $\mathcal{L}_{A}$-subharmonic on $\mathbb{G}$. By the submean condition (2.9) for the sub-Laplacian $\mathcal{L}_{A}$, this is equivalent to $u(x) \leq M_{r}^{\mathcal{L}_{A}}(u)(x)$, for every $x \in \mathbb{G}$ and every $r>0$. Thanks to identity (3.6) in Lemma 3.4 by taking $x=T_{A}(\xi)$ and $r=\rho c_{A}^{1 /(2-Q)}$ (with $\xi \in \mathbb{G}$ and $\rho>0$ arbitrary), this rewrites as

$$
u\left(T_{A}(\xi)\right) \leq M_{\rho}\left(u \circ T_{A}\right)(\xi), \quad \text { for every } \xi \in \mathbb{G} \text { and } \rho>0 .
$$

Since $u \circ T_{A}: \mathbb{G} \rightarrow[-\infty, \infty)$ is clearly u.s.c., the last inequality is equivalent to the subharmonicity of $u \circ T_{A}$ w.r.t. the sub-Laplacian $\Delta_{\mathbb{G}}$.

The reverse implication can be proved analogously.

Before proving our main result, Theorem 3.8 below, we seize the opportunity to remove any ambiguity from the notation $A \mapsto T_{A}$ (which is a multiple-valued map) once and for all by introducing new notation. 
Definition 3.7. Let $\mathbb{G}$ be a free homogenous Carnot group $\mathbb{G}=\left(\mathbb{R}^{N}, *, \delta_{\lambda}\right)$ with $m$ generators. Let $Z_{1}, \ldots, Z_{N}$ be the basis of $\operatorname{Lie}(\mathbb{G})$ of the left-invariant vector fields coinciding at the origin with $\left.\partial_{x_{1}}\right|_{0}, \ldots,\left.\partial_{x_{N}}\right|_{0}$, respectively.

Given any real non-singular matrix $B=\left(b_{i, j}\right)_{i, j \leq m}$ we denote by $\varphi_{B}$ the unique Lie-algebra isomorphism of $\operatorname{Lie}(\mathbb{G})$ mapping $Z_{i}$ into $\sum_{j=1}^{m} b_{i, j} Z_{j}$, for every $i \in$ $\{1, \ldots, m\}$. With this position, we let

$$
\Theta_{B}:=\operatorname{Exp} \circ \varphi_{B} \circ \log ;
$$

that is, $\Theta_{B}$ is the (unique) Lie-group automorphism of $\mathbb{G}$ whose differential is $\varphi_{B}$.

Theorem 3.8. Let $\mathbb{G}$ be a free (homogenous) Carnot group with $m$ generators, and fix the sub-Laplacian $\Delta_{\mathbb{G}}=\sum_{i=1}^{m} Z_{i}^{2}$ related to the left-invariant vector fields $Z_{1}, \ldots, Z_{m}$ coinciding at the origin with $\left.\partial_{x_{1}}\right|_{0}, \ldots,\left.\partial_{x_{m}}\right|_{0}$.

Let $u: \mathbb{G} \rightarrow[-\infty, \infty)$ be upper semicontinuous. Then the following facts are equivalent (here $\Theta_{B}$ is as in Definition 3.7):

(i) $u$ is v-convex on $\mathbb{G}$;

(ii) $u \circ \Theta_{B}$ is subharmonic on $\mathbb{G}$ with respect to $\Delta_{\mathbb{G}}$, for every real $m \times m$ non-singular matrix $B$;

(iii) $u \circ \Theta_{B}$ is subharmonic on $\mathbb{G}$ with respect to $\Delta_{\mathbb{G}}$, for every real $m \times m$ symmetric and positive definite matrix $B$.

Proof. It follows by collecting together Proposition 3.5 and the very definition of v-convexity. We have indeed the following implications.

(i) $\Rightarrow$ (ii): Let $u$ be v-convex and let $B \in \mathcal{M}$ be non-singular. Consider the matrix $A:=B^{T} \cdot B$. Clearly $A \in \mathcal{A}$ and note that $\Theta_{B}$ is one of the admissible maps $T_{A}$ introduced in Lemma 3.1. Since $u$ is v-convex, it is in particular $\mathcal{L}_{A}$-subharmonic. Now, assertion (1) in Remark 3.6 ensures that $u \circ T_{A}=u \circ \Theta_{B}$ is $\Delta_{\mathbb{G}}$-subharmonic.

(ii) $\Rightarrow$ (iii): Obvious.

(iii) $\Rightarrow($ i): Suppose that hypothesis (iii) holds. We aim to prove that $u$ is v-

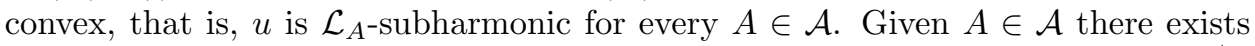
a unique symmetric positive definite matrix $B$ such that $A=B^{2}$, say $B=A^{1 / 2}$. By hypothesis (iii), $u \circ \Theta_{A^{1 / 2}}$ is $\Delta_{\mathbb{G}^{-}}$subharmonic. Note that $\Theta_{A^{1 / 2}}$ is one of the admissible maps $T_{A}$ introduced in Lemma 3.1, since $B^{T} \cdot B=B^{2}=A$. Now, assertion (2) in Remark 3.6 ensures that $u$ is $\mathcal{L}_{A}$-subharmonic, as we intended to prove. This completes the proof of the theorem.

\section{REFERENCES}

1. Z.M. Balogh, M. Rickly, Regularity of convex functions on Heisenberg groups, Ann. Sc. Norm. Super. Pisa, Cl. Sci. 2 (2003), 847-868. MR2040646 (2006d:43006)

2. A. Bonfiglioli, Lifting of convex functions on Carnot groups and lack of convexity for a gauge function, Archiv der Math. 93 (2009), 277-286. MR2540794 (2010j:22011)

3. A. Bonfiglioli, E. Lanconelli, Subharmonic functions on Carnot groups, Math. Ann. 325 (2003), 97-122. MR1957266 (2004b:35047)

4. A. Bonfiglioli, E. Lanconelli, F. Uguzzoni, Uniform Gaussian estimates of the fundamental solutions for heat operators on Carnot groups, Adv. Differential Equations 7 (2002), 11531192. MR.1919700 (2003f:35054)

5. A. Bonfiglioli, E. Lanconelli, F. Uguzzoni, Stratified Lie Groups and Potential Theory for Their Sub-Laplacians, Springer Monographs in Mathematics 26, Springer, New York, 2007. MR 2363343 (2009m:22012)

6. A. Bonfiglioli, F. Uguzzoni, Families of diffeomorphic sub-Laplacians and free Carnot groups, Forum Math. 16 (2004), 403-415. MR2050190(2005f:22029) 
7. L. Capogna, D. Maldonado, A note on the engulfing property and the $\Gamma^{1+\alpha}$-regularity of convex functions in Carnot groups, Proc. Amer. Math. Soc. 134 (2006), 3191-3199. MR 2231902 (2007e:35033)

8. L. Capogna, S.D. Pauls, J.T. Tyson, Convexity and horizontal second fundamental forms for hypersurfaces in Carnot groups, Trans. Amer. Math. Soc. 362 (2010), 4045-4062. MR 2608394 (2011d:43007)

9. H. Dah-Yan, Some interpolations and refinements of Hadamard's inequality for $r$-convex functions in Carnot groups, Math. Inequal. Appl. 10 (2007), 287-297. MR2312084(2008h:26004)

10. D. Danielli, N. Garofalo, D.-M. Nhieu, Notions of convexity in Carnot groups, Comm. Anal. Geom. 11 (2003), 263-341. MR2014879 (2004m:22014)

11. D. Danielli, N. Garofalo, D.-M. Nhieu, F. Tournier, The theorem of Busemann-FellerAlexandrov in Carnot groups, Commun. Anal. Geom. 12 (2004), 853-886. MR 2104079 (2005j:22005)

12. G.B. Folland, Subelliptic estimates and function spaces on nilpotent Lie groups, Ark. Mat. 13 (1975), 161-207. MR0494315 (58:13215)

13. L. Gallardo, Capacités, mouvement brownien et problème de l'épine de Lebesgue sur les groupes de Lie nilpotents, Lectures Notes in Math. 928, Proc. VII Oberwolfach conference on probability measures on groups, Springer-Verlag, Berlin-New York, 1982. MR669065 (84a:60089)

14. N. Garofalo, Geometric second derivative estimates in Carnot groups and convexity, Manuscr. Math. 126 (2008), 353-373. MR.2411233 (2009i:35049)

15. N. Garofalo, F. Tournier, New properties of convex functions in the Heisenberg group, Trans. Amer. Math. Soc. 358 (2006), 2011-2055. MR2197446 (2006k:26017)

16. M. Grayson, R. Grossman, Models for free nilpotent Lie algebras, J. Algebra 135 (1990), 177-191. MR1076084 (91g:17015)

17. C.E. Gutiérrez, A. Montanari, Maximum and comparison principles for convex functions on the Heisenberg group, Comm. Partial Differential Equations 29 (2004), 1305-1334. MR 2103838(2005h:35024)

18. C.E. Gutiérrez, A. Montanari, On the second order derivatives of convex functions on the Heisenberg group, Ann. Sc. Norm. Super. Pisa Cl. Sci. (5) 3 (2004), 349-366. MR2075987 (2005f:26038)

19. M. Hall, A basis for free Lie rings and higher commutators in free groups, Proc. Amer. Math. Soc. 1 (1950), 575-581. MR0038336(12:388a)

20. L. Hörmander, Notions of Convexity, Progress in Mathematics 127, Birkhäuser, Boston, 1994. MR.1301332 (95k:00002)

21. P. Juutinen, G. Lu, J.J. Manfredi, B. Stroffolini, Convex functions on Carnot groups, Rev. Mat. Iberoam. 23 (2007), 191-200. MR2351130(2008m:49165)

22. G. Lu, J.J. Manfredi, B. Stroffolini, Convex functions on the Heisenberg group, Calc. Var. Partial Differential Equations 19 (2004), 1-22. MR2027845 (2004m:35088)

23. V. Magnani, Lipschitz continuity, Aleksandrov theorem and characterizations for $H$-convex functions, Math. Ann. 334 (2006), 199-233. MR2208954 (2007c:49005)

24. R. Monti, M. Rickly, Geodetically convex sets in the Heisenberg group, J. Convex Anal. 12 (2005), 187-196. MR2135806 (2005m:53045)

25. M. Rickly, First-order regularity of convex functions on Carnot groups, J. Geom. Anal. 16 (2006), 679-702. MR 2271949 (2008a:43007)

26. M. Sun, X. Yang, Generalized Hadamard's inequality and r-convex functions in Carnot groups, J. Math. Anal. Appl. 294 (2004), 387-398. MR2061332(2005a:26037)

27. M. Sun, X. Yang, Inequalities of Hadamard type for $r$-convex functions in Carnot groups, Acta Math. Appl. Sin. Engl. Ser. 20 (2004), 123-132. MR.2052711(2005f:22010)

28. M. Sun, X. Yang, Lipschitz continuity for H-convex functions in Carnot groups, Commun. Contemp. Math. 8 (2006), 1-8. MR2208809 (2007c:43009)

29. M. Sun, X. Yang, Quasi-convex functions in Carnot groups, Chin. Ann. Math., Ser. B, 28 (2007), 235-242. MR2316661 (2009d:43033)

30. V.S. Varadarajan, Lie Groups, Lie Algebras and Their Representations, Graduate Texts in Mathematics 102, Springer-Verlag, New York, 1984. MR746308 (85e:22001)

31. N.T. Varopoulos, L. Saloff-Coste, Th. Coulhon, Analysis and Geometry on Groups, Cambridge Tracts in Mathematics 100, Cambridge University Press, Cambridge, 1992. MR 1218884 (95f:43008) 
32. C. Wang, Viscosity convex functions on Carnot groups, Proc. Amer. Math. Soc. 133 (2005), 1247-1253. MR2117228 (2006a:58026)

33. F.W. Warner, Foundations of Differentiable Manifolds and Lie Groups, Graduate Texts in Mathematics 94, Springer-Verlag, New York-Berlin, 1983. MR722297 (84k:58001)

Dipartimento di Matematica, Università degli Studi di Bologna, Piazza di Porta San Donato, 5, 40126 Bologna, Italy

E-mail address: bonfigli@dm.unibo.it

Dipartimento di Matematica, Università degli Studi di Bologna, Piazza di Porta San Donato, 5, 40126 Bologna, Italy

E-mail address: lanconel@dm.unibo.it 